\title{
Crystallite Size and Microstrain Measurement of Cathode Material after Mechanical Milling using Neutron Diffraction Technique
}

\author{
A. Fajar ${ }^{*}$, Gunawan, E. Kartini, H. Mugirahardjo and M. Ihsan \\ Center for Technology of Nuclear Industry Materials, National Nuclear Energy Agency \\ Puspiptek, Serpong, Tangerang 15314, Indonesia
}

\author{
ARTICLE INFO \\ Article history: \\ Received 7 October 2010 \\ Received in Revised form 31 October 2010 \\ Accepted 1 November 2010 \\ Keywords: \\ Cobalt compound \\ Crystallite size \\ $\mathrm{LiCoO}_{2}$ \\ Lithium compounds \\ Microstrain \\ Rietveld refinement
}

\begin{abstract}
A B S T R A C T
The measurements of neutron diffraction patterns of commercially product and 10 hour mechanically milled cathode material lithium cobaltites $\left(\mathrm{LiCoO}_{2}\right)$ have been performed. Rietveld analysis using FullProf does not show the change of crystal structure due to milling process, but the diffraction pattern has a lower intensity and the diffraction-line was broadening. The results of line-broadening study on milled sample using Rietveld methods detected that the microstrain was occurred in the sample with value $0.127080(35) \%$ and the average crystallite size was $424.78(20) \AA$.
\end{abstract}

(C) 2010 Atom Indonesia. All rights reserved

\section{INTRODUCTION}

The lithium ion rechargeable battery is one of the advanced power sources with a leading position in miniatures batteries because of its high energy density [1,2]. This battery utilizes lithium host materials in which lithium ions intercalate and deintercalate into/from the anode and the cathode materials. The materials being examined as cathodes are the layered transition metal oxides, such as $\mathrm{LiCoO}_{2}$ and the oxides having a spinel structure such as $\mathrm{LiMn}_{2} \mathrm{O}_{4}$. Of these, $\mathrm{LiCoO}_{2}$ has been examined widely and was commercialized for lithium ion batteries although cobalt is more expensive and less intimate to environment than manganese oxides [3]. $\mathrm{LiCoO}_{2}$ structure is based on a close-packed network of oxygen atoms with the $\mathrm{Li}^{+}$and $\mathrm{Co}^{3+}$ ions ordering on alternation (111) planes of the cubic rock salt structure. When the cell is charging, the lithium ion deintercalate from the $\mathrm{LiCoO}_{2}$ structure. When the cell is discharging, the lithium ions intercalate into the $\mathrm{Li}_{1-\mathrm{x}} \mathrm{CoO}_{2}$ structure.

Although Li-ion batteries are attractive power-storage devices that have high energy density, their high power density is generally low due to the high level of polarization at higher current rates. In this regard nano-sized or nano-structured active materials have been widely synthesized in order to resolve the problems because nanomaterial

\footnotetext{
* Corresponding author.

E-mail address: andika@batan.go.id (A. Fajar)
}

has very large reaction area and short diffusion length for $\mathrm{Li}^{+}$, resulting in high capacity, high power, enhanced structural stability and longer cycle life [4]. Mechanical milling has been used extensively to synthesize nanocrystalline materials. This is essentially due to the fact that mechanical milling is a very simple room temperature technique that is capable of producing nanocrystalline structures in almost any type of material and scaling up of this technique to produce tonnage quantities on an industrial scale has already been proven [5].

In this study, mechanical High Energy Milling was employed to $\mathrm{LiCoO}_{2}$ powder to obtain nano-sized powder. Neutron diffraction measurements have been performed to examine the effect of mechanical milling. The crystallite size (size of the coherently diffracting domains, often equated with the grain size) and lattice strain which are the important parameters in mechanically milled powders were determined by measuring the broadening of the diffraction lines using the whole pattern analysis Rietveld method.

\section{Rietveld refinement}

Rietveld refinement is becoming more popular for nonstructural applications, such as texture [6] and residual-stress [7] determination. It is practice to estimate domain size and strain values from the refined profile width parameters. An accurate modeling of line width is a necessary 
prerequisite for microstructure study. We used the Rietveld refinement program contained in the FullProf suite [8] for analysis. The microstructures effects within FullProf are treated using the Voigt approximation: Both instrumental and sample intrinsic profiles are supposed to be described approximately by a convolution of Lorentzian and Gaussian components. The Thompson-CoxHastings (TCH) pseudo-Voigt profile function is used to mimic the exact Voigt function and it includes the Finger's treatment of the axial divergence. The full width at half maximum (FWHM) of the Gaussian $\left(\mathrm{H}_{\mathrm{G}}\right)$ and Lorentzian $\left(\mathrm{H}_{\mathrm{L}}\right)$ components of the line profile have an angular dependence given by:

$$
\begin{aligned}
H_{G}^{2} & =U \tan ^{2} \theta+V \tan \theta+W+I_{G} / \cos ^{2} \theta \\
\text { and } & \\
H_{L} & =X \tan \theta+Y / \cos \theta+Z
\end{aligned}
$$

Here, $U, V, W, X, Y, I_{G}$ and $Z$ are refinable parameters. Equation (2) is the Lorentzian line width and includes contributions from Lorentzian size broadening $X$ and Lorentzian strain broadening $Y ; Z$ is customarily set to zero. In the case of a constant wavelength experiment the broadening of reflections due to microstrains has an angular dependence of the form:

$$
\begin{gathered}
\Delta d / d=F W(s) \cot \theta=\text { constant } \\
\text { or } \\
F W(s)=(\Delta d / d) \tan \theta=\varepsilon \tan \theta
\end{gathered}
$$

The corresponding dependence for the size effect is given by the Scherrer formula:

$$
\begin{gathered}
\Delta d / d^{2}=F W(S) \cot \theta / d=\text { constant } \\
\text { or } \\
F W(s)=\lambda\left(\Delta d / d^{2}\right) / 2 \cos \theta
\end{gathered}
$$

where $\lambda$ is the wavelength. Hence, it is easy to recognize from equations (1) and (2) that parameters size and strain broadening, are related to component $Y$ and $I_{G}$, and component $X$ and $U$, respectively as shown in Table 1.

Table 1. Parameters size and strain from Gaussian and Lorentzian.

\begin{tabular}{lcc}
\hline Parameters & $\begin{array}{c}\text { Gaussian } \\
\text { component }\end{array}$ & $\begin{array}{c}\text { Lorentzian } \\
\text { component }\end{array}$ \\
\hline Size $(\AA)$ & $\frac{180 K \lambda}{\pi \sqrt{I_{G}}}$ & $\frac{180 K \lambda}{\pi Y}$ \\
Strain $(\%)$ & $\left(\frac{\pi}{1.8}\right) \sqrt{U-U_{\text {ins }}}$ & $\left(\frac{\pi}{1.8}\right) X$ \\
\hline
\end{tabular}

Instrument introduces some amount of broadening that has to be properly corrected in order to obtain reliable information about domain size and strain from line broadening of the sample under investigation [9].

\section{EXPERIMENTAL METHODS}

The sample used in this study is lithium cobaltite $\left(\mathrm{LiCoO}_{2}\right)$ powder obtained commercially from Aldrich. The mechanical milling was carried out in a High Energy Milling machine (HEM, PW700i). The $\mathrm{LiCoO}_{2}$ powder was poured into a stainless steel container together with stainless steel balls without controlled atmosphere. The weight ratio of ball-to-powder was 2:1. The $\mathrm{LiCoO}_{2}$ sample was milled at $1300 \mathrm{rpm}$ for 10 hour.

Neutron powder diffraction data were collected from as-received $\mathrm{LiCoO}_{2}$ and after 10 hour milling using a high resolution neutron powder diffractometer (DN3) installed at the Neutron Scattering Laboratory, Indonesia National Nuclear Energy Agency (BATAN). A hot-pressed Ge (331) monochromator with an $89^{\circ}$ take-off angle yielding a wavelength of $1.8223 \AA$ was used. The sample was loaded into a $12 \mathrm{~mm}$ diameter vanadium can. Patterns were collected at ambient temperature over the $2 \theta$ range of $10-150^{\circ}$ with a step size of $0.05^{\circ}$.

The instrumental broadening was characterized by measuring the standard reference material SRM640b Si from the National Institute of Standards and Technology (NIST) that shows a minimal amount of physical line broadening caused by defects and small crystallite size. The result was compared with the diffraction-line broadening of asreceived $\mathrm{LiCoO}_{2}$ powder whether the as-received sample can be used as the instrumental standard sample in the analysis [10].

The analysis of diffraction-line broadening was performed using FullProf by the Voigt approximation as the initial parameter described in the previous paper [11]. An input file containing the instrumental resolution function (IRF) is provided from $\mathrm{Si}$ and as-received $\mathrm{LiCoO}_{2}$ sample to get the new file containing information about the microstructures. The analysis were performed by fixing $V$ and $W$ to zero, and refine the rest of parameters in above formula which has a meaning in terms of strains $(U, X)$ or size $\left(Y, I_{G}\right)$. We also performed the analysis using Williamson-Hall plot which is usually used in the X-ray diffraction analysis in order to compare the above results. 


\section{RESULTS AND DISCUSSION}

Figures 1(a) and (b) show the results of Rietveld refinement for both as-received $\mathrm{LiCoO}_{2}$ and the $\mathrm{LiCoO}_{2}$ after 10 hour milling. Both samples were measured in the same conditions. The calculated profile is in a good agreement with the observed data. From the diffraction data it is known that the milling process until 10 hour do not change the crystal structure since there is no new diffraction peak position observed as shown in Fig. 1(b). But as can be seen that the peaks in the diffraction pattern become broader and their intensity decreased in milled sample compare to as-received sample. It should be due to the contribution of crystallite size and microstrain changes in the sample which will be explained below.

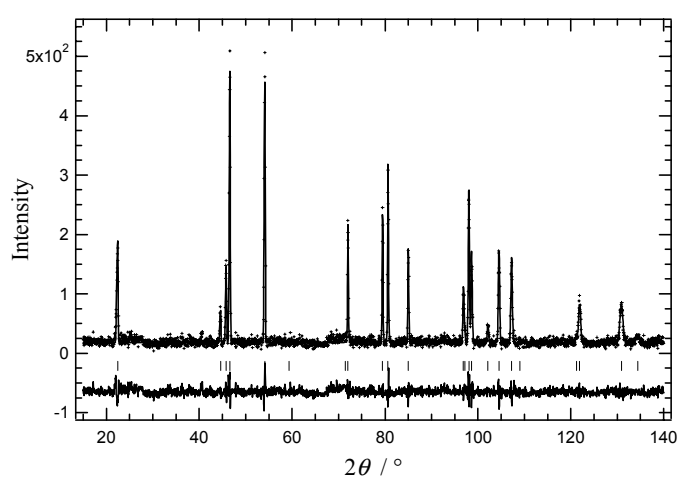

(a)

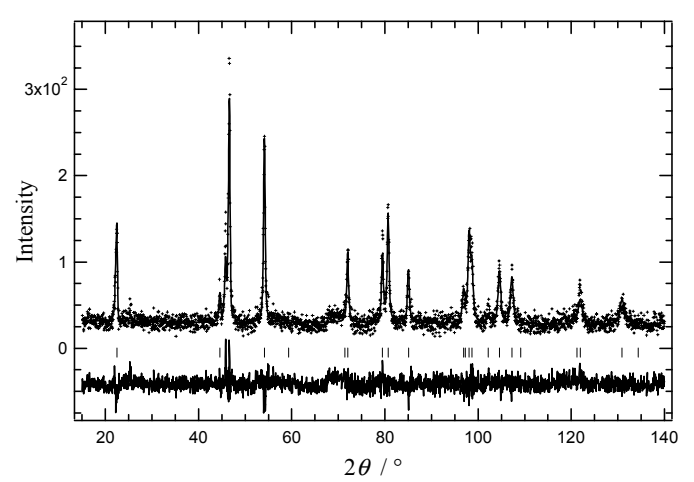

(b)

Fig. 1. Rietveld refinements using neutron diffraction data for commercial $\mathrm{LiCoO}_{2}$ powder, (a) as-received and (b) after 10 hour ball milling. The plus markers are the data and the line through the markers is the result from the refinement. The Bragg peak positions are shown as short vertical lines. At the bottom is shown the difference between the data and the calculated profile.

The refined structural parameters are similar for both samples as summarized in Table 2. The small change in lattice parameters was obtained due to the milling process. The difference of the reliability factor $R$ reflects the differences in signal to background ratios.

Table 2. Refined structural parameters of as-received $\mathrm{LiCoO}_{2}$ sample and after 10 hour milling from neutron diffraction data using FullProf.

\begin{tabular}{|c|c|c|}
\hline \multirow{3}{*}{$\begin{array}{l}\text { Space group } \\
\text { Crystal system }\end{array}$} & As received & After $10 \mathrm{~h}$ milled \\
\hline & \multicolumn{2}{|c|}{$R-3 m(\mathrm{~A}-166)$} \\
\hline & \multicolumn{2}{|c|}{ Trigonal/rhombohedral } \\
\hline$a=b(\AA)$ & $2.81622(1)$ & $2.81534(3)$ \\
\hline$c(\AA)$ & $14.053111(3)$ & $14.054284(8)$ \\
\hline$\alpha=\beta \cdot \gamma$ & $90: 120$ & $90: 120$ \\
\hline$V\left(\AA^{3}\right)$ & $96.524(5)$ & $96.472(13)$ \\
\hline $\mathrm{Li}, 3 b(0 ; 0 ; 0,5)$ & & \\
\hline Occ. Fac. & 1 & 1 \\
\hline$B_{\text {iso }}\left(\AA^{2}\right)$ & $0.25(23)$ & $0.66(36)$ \\
\hline Co, 3a $(0 ; 0 ; 0)$ & & \\
\hline Occ.Fac. & 1 & 1 \\
\hline$B_{\text {iso }}\left(\AA^{2}\right)$ & $0.07(17)$ & $0.53(29)$ \\
\hline $0,6 c(0 ; 0 ; z)$ & & \\
\hline$z$ & $0.2606(2)$ & $0.2609(3)$ \\
\hline Occ.Fac. & 1 & 1 \\
\hline$B_{\text {iso }}\left(\AA^{2}\right)$ & $0.09(8)$ & $0.39(12)$ \\
\hline$R(\%)$ & 16.5 & 14.2 \\
\hline$w R(\%)$ & 21.8 & 17.7 \\
\hline $\operatorname{red} \cdot \chi^{2}$ & 1.27 & 1.21 \\
\hline
\end{tabular}

Figure 2 shows the full width at halfmaximum (FWHM) for all available Bragg reflections for as-received, after 10 hour milled $\mathrm{LiCoO}_{2}$ and NBS SRM640b $\mathrm{Si}$ samples as determined from diffraction pattern using Rietveld refinements. As mentioned above milled powder sample has broader line profile. The NBS SRM640b $\mathrm{Si}$ powder slightly shows smaller value than as-received $\mathrm{LiCoO}_{2}$ only in the low scattering angle and at higher angle is almost similar. Therefore, the as-received $\mathrm{LiCoO}_{2}$ may be used as the standard sample to correct the instrumental effects in $10 \mathrm{~h}$ hour milled sample.

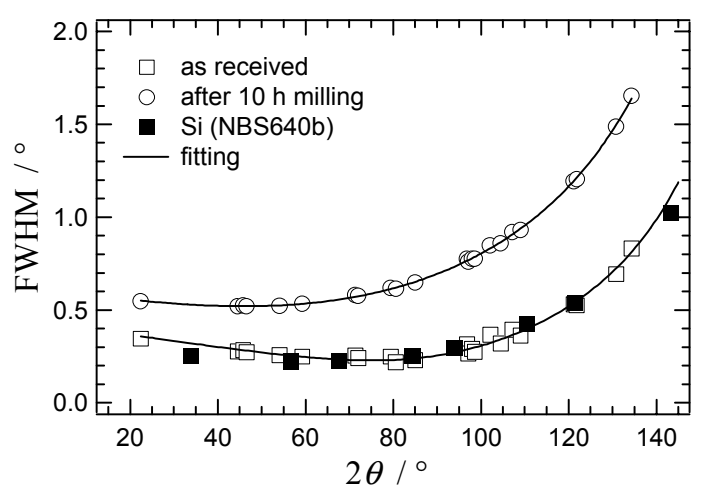

Fig. 2. FWHM versus diffraction angle as obtained from HRPD measurements, for three samples; $\mathrm{LiCoO} 2$ as received, after $10 \mathrm{~h}$ milling and NBS640b Si. 
The intrinsic (instrumental) line width is an important parameter in line-broadening analysis. It is advantageous that the physical broadening, which contains microstructures information, be more pronounced than the instrumental broadening [9]. In Figure 3, we show 104 diffraction lines for both as-received and milled samples. It is evident that the ratio of broadening changes by an order of magnitude.

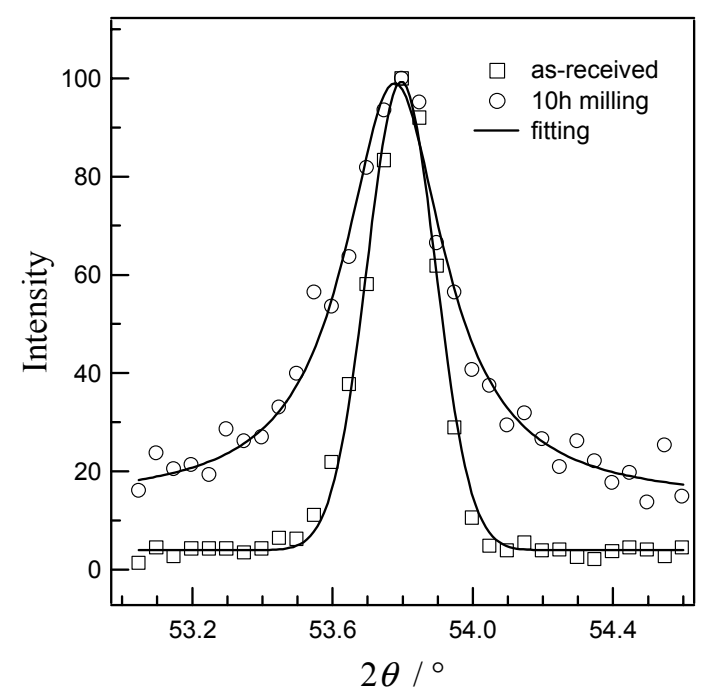

Fig. 3. (104) diffraction lines of $\mathrm{LiCoO}_{2}$ powder samples (a) as-received and (b) after 10 hour ball milling, normalized to same maximum peak height.

The crystallite size and microstrain obtained from the FullProf refinement by refining various combinations of parameters is summarized in Table 2. As expected, only strain or size can be determined if only one parameter is refined. Then both size and microstrain can be obtained only if we refine simultaneously either Lorentzian size and strain components or both Gaussian components, but not the combination of them, such as $I_{G^{-}} Y, U-X$, $I_{G}-X$ etc. The standard deviations appearing in the global average apparent size and strain are calculated using the different reciprocal lattice directions. It is a measure of the degree of anisotropy, not of the estimated error. The crystallite size become higher and the strain lower if both Lorentzian and Gaussian components were refined simultaneously. But the results are quite different for refinement of each component. If the $\chi^{2}$ value was considered the refinement using $X$ and $Y$ parameters give a good agreement than $U$ and $I_{G}$ parameters. Therefore, it is considered that the crystallite size of $10 \mathrm{~h}$ milled $\mathrm{LiCoO}_{2}$ powder is $424.78(20) \AA$ and the strain is $0.127080(35) \%$ as shown in Table 3.
Table 3. Crystallite size and microstrain of $\mathrm{LiCoO}_{2}$ after 10 hour milling

\begin{tabular}{cccc}
\hline Parameters & Red. $\chi^{2}$ & $\begin{array}{c}\text { Average } \\
\text { apparent size } \\
(\AA)\end{array}$ & $\begin{array}{c}\text { Average } \\
\text { maximum strain } \\
(\%)\end{array}$ \\
\hline$U$ & 1.47 & - & $0.344011(207)$ \\
$X$ & 1.24 & - & $0.318423(133)$ \\
$I_{G}$ & 1.41 & $237.24(3)$ & - \\
$Y$ & 1.22 & $257.70(7)$ & - \\
$U$ and $I_{G}$ & 1.23 & $277.46(4)$ & $0.181733(155)$ \\
$X$ and $Y$ & 1.21 & $424.78(20)$ & $0.127080(35)$ \\
\hline
\end{tabular}

The determination of average apparent size and average maximum strain have been performed for the as-received $\mathrm{LiCoO}_{2}$ sample. The IRF file was provided from the FHWM value of $\mathrm{Si}$ sample. In this case the refinement by using two parameters cannot be performed. By considering the lowest $\chi^{2}$ value, we obtained that the crystallite size is 4269.84(1.31) $\AA$ by refining $\mathrm{Y}$ parameters and the microstrain is $0.002802(1) \%$ by refining $X$ parameter. From this result we know that the milling process reduces the crystallite size smaller and produce the microstrain in the sample.

Here, the Williamson-Hall method will be described for determining the crystallite size and microstrain in order to make comparison with the results obtained from the whole pattern refinements. They suggested that the broadening due to size and microstrain can be expressed as follows [11],

$$
\begin{gathered}
F W(s)=\frac{K \lambda}{L \cos \theta}+\eta \tan \theta \\
\text { or } \\
F W(s) \cos \theta=\frac{K \lambda}{L}+4 \varepsilon \sin \theta
\end{gathered}
$$

where L is crystallite size $(\AA)$ and $\varepsilon$ is average maximum microstrain. If we plot $F W(s) \cos \theta$ according to $\sin \theta$, the interception of fitting line with vertical axis equal to the inverse of the size and a slope equal to the value of the microstrain. From the interception of fitting line with vertical axis we can determine the crystallite size and from the slope of line we calculate the microstrain. Figure 4 shows the Williamson-Hall obtain from the diffraction pattern of (a) as-received $\mathrm{LiCoO}_{2}$ sample without instrumental broadening correction and (b) 10 hour milled sample with correction using as-received $\mathrm{LiCoO}_{2}$ data. From Fig. 4(a) the crystallite size and microstrain can not be determined correctly since no correction data is applied. But this method makes it possible to obtain a qualitative mean value characterizing each of the effects that cause the increase in peak broadening. In the case of Fig. 4(a) the slope of fitting line is almost horizontal, it means that the crystal sample contain a small amount of 
microstrain. On the other hand Fig. 4(b) describe that the strain value is quite significant. From the interception and slope, it is obtained that the crystallite size is $658.68 \AA$ and the microstrain is $0.092058 \%$.

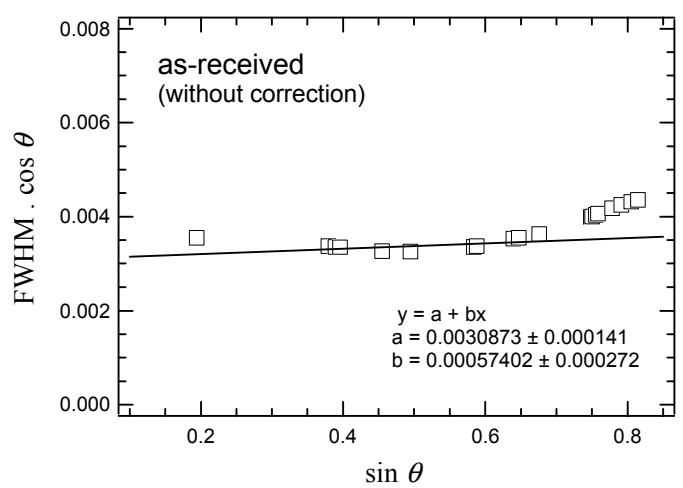

(a)

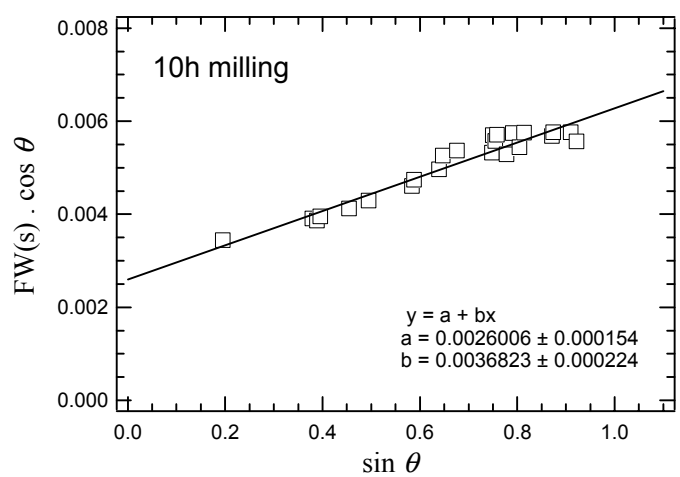

(b)

Fig. 4. Williamson-Hall plot obtained by using profile fitting of the neutron diffraction pattern for commercial $\mathrm{LiCoO}_{2}$ powder, (a) as-received and (b) after 10 hour ball milling.

These values are closer to the results of Rietveld refinements by refining the Lorentzian components. Since this method works by considering both the limited size of the crystals and the presence of crystallographic distortions lead to Lorentzian intensity distribution [12].

\section{CONCLUSIONS}

The line-broadening analysis obtained by Rietveld methods on neutron diffraction pattern collected from neutron diffractometer DN3 has been described. The main problem in this line-broadening analysis seems to be the size-strain separation and the characterization of instrumental broadening. The mechanical milling process makes the crystallite size to be smaller and produce the microstrain in the sample. The average apparent domain sizes and microstrain of 10 hour milled $\mathrm{LiCoO}_{2}$ powder sample obtained by refining Lorentzian size and strain broadening are $424.78(20) \AA$ and $0.127080(35) \%$. In this sample we can not refine the Lorentzian components together with the Gaussian components. The Williamson-Hall method has been also performed in order to determine crystallite size and microstrain for comparison, and the results are closer to the above values.

\section{REFERENCES}

1. J.M. Tarascon and M. Armand, Nature 414 (2001) 356.

2. M. Wakihara, Mater. Sci. Engin. $\mathbf{R 3 3}$ (2001) 109.

3. P.J. Cho, E.D. Jeong and Y.B. Shim, Bull. Korean Chem. Soc. 19 (1998) 39.

4. M. Jo, Y.S. Hong, J. Choo and J. Cho., J. Electrochem. Soc. 156 (2009) A430.

5. C. Suryanarayana, Mechanical Alloying and Milling, Marcel Dekker, New York (2004).

6. R.B. Von Dreele, J. Appl. Cryst. 30 (1997) 517.

7. N.C. Popa and D. Balzar, J. Appl. Cryst. 34 (2001) 187.

8. J.R. Carjaval, Physica B 192 (1993) 55.

9. D. Balzar, N. Audebrand, M.R. Daymond, A. Fitch, A. Hewat, J.I. Langford, A. Le Bail, D. Loue, O. Masson, C.N. McCowan, N.C. Popa, P.W. Stephens, and B. H. Toby, J. Appl. Cryst. 37 (2004) 911.

10. A. Fajar and H. Mugirahardjo, Atom Indonesia 36 (2010) 1.

11. A. Fajar, Gunawan, E. Kartini, H. Mugirahardjo, A. Purwanto and Y. Sarwanto, Ind. J. Mat. Sci., December Special Edition (2009) 35.

12. R. Guinebretiere, X-ray Diffraction by Polycrystalline Materials ISTE Ltd., UK (2007). 